\title{
Viscosity iteration algorithm for a $\varrho$-strictly pseudononspreading mapping in a Hilbert space
}

\author{
Bin-Chao Deng, Tong Chen and Zhi-Fang Li*
}

\section{"Correspondence:}

Izf399@yahoo.com.cn

School of Management, Tianjin

University, Tianjin, 300072, China

\begin{abstract}
In this paper, we discuss the strong convergence of the viscosity approximation method in Hilbert spaces relatively to the computation of fixed points of an operator in $\varrho$-strictly pseudononspreading. Under suitable conditions, some strong convergence theorems are proved. Our work improves previous results for nonspreading mappings.
\end{abstract}

Keywords: nonspreading mapping; $\varrho$-strictly pseudononspreading; demicontractive; fixed point; quasi-nonexpansive

\section{Introduction}

Throughout this paper, we always assume that $H$ is a real Hilbert space endowed with an inner product and its induced norm denoted by $\langle\cdot, \cdot\rangle$ and $|\cdot|$, respectively. Let $C$ be a nonempty, closed and convex subset of $H$ and let $A: C \rightarrow H$ be a nonlinear mapping.

Definition 1.1 $A$ is said to be

(i) monotone if

$$
\langle A x-A y, x-y\rangle \geq 0, \quad \forall x, y \in C
$$

(ii) strongly monotone if there exists a constant $\alpha>0$ such that

$$
\langle A x-A y, x-y\rangle \geq \alpha\|x-y\|^{2}, \quad \forall x, y \in C .
$$

For such a case, $A$ is said to be $\alpha$-strongly-monotone;

(iii) inverse-strongly monotone if there exists a constant $\alpha>0$ such that

$$
\langle A x-A y, x-y\rangle \geq \alpha\|A x-A y\|^{2}, \quad \forall x, y \in C .
$$

For such a case, $A$ is said to be $\alpha$-inverse-strongly-monotone;

(iv) $k$-Lipschitz continuous if there exists a constant $k \geq 0$ such that

$$
\|A x-A y\| \leq k\|x-y\|, \quad \forall x, y \in C .
$$


Remark 1.2 Let $F=\mu B-\gamma f$, where $B$ is a $\theta$-Lipschitz and $\eta$-strongly monotone operator on $H$ with $\theta>0$ and $f$ is a Lipschitz mapping on $H$ with coefficient $L>0,0<\gamma \leq \frac{\mu \eta}{L}$. It is a simple matter to see that the operator $F$ is $(\mu \eta-\gamma L)$-strongly monotone over $H$, i.e.,

$$
\langle F x-F y, x-y\rangle \geq(\mu \eta-\gamma L)\|x-y\|^{2}, \quad \forall(x, y) \in H \times H .
$$

The classical variational inequality, which is denoted by $\operatorname{VI}(A, C)$, is to find $x \in C$ such that

$$
\langle A x, y-x\rangle \geq 0, \quad \forall y \in C .
$$

The variational inequality has been extensively studied in literature (see [1-7] and the references therein).

A mapping $T: C \rightarrow C$ is said to be nonexpansive if

$$
\|T x-T y\| \leq\|x-y\|, \quad \forall x, y \in C .
$$

A mapping $T$ is said to be firmly nonexpansive if

$$
\|T x-T y\|^{2} \leq\langle x-T x, y-T y\rangle, \quad \forall x, y \in C ;
$$

see, for instance, [8-11]. It is known that a mapping $T: C \rightarrow C$ is firmly nonexpansive if and only if

$$
\|T x-T y\|^{2}+\|(I-T) x-(I-T) y\|^{2} \leq\|x-y\|^{2}, \quad \forall x, y \in C .
$$

$T$ is said to be nonspreading in [12] if

$$
\|T x-T y\|^{2} \leq\|T x-y\|^{2}+\|T y-x\|^{2}, \quad \forall x, y \in C .
$$

It is shown in [13] that (1.2) is equivalent to

$$
\|T x-T y\|^{2} \leq\|x-y\|^{2}+2\langle x-T x, y-T y\rangle, \quad \forall x, y \in C .
$$

These mappings are generalization of a firmly nonexpansive mapping in a Hilbert space. $T: C \rightarrow C$ is said to be firmly nonexpansive if

$$
\|T x-T y\|^{2} \leq\langle x-y, T x-T y\rangle, \quad \forall x, y \in C .
$$

See [14-17] for more information on firmly nonexpansive mappings.

Definition 1.3 $T: H \rightarrow H$ is called demicontractive on $H$ if there exists a constant $\alpha<1$ such that

$$
\|T x-q\|^{2} \leq\|x-q\|^{2}+\alpha\|x-T x\|^{2}, \quad \forall(x, q) \in H \times F_{i x}(T) .
$$


Definition 1.4 [18] $T: D(T) \subseteq H \rightarrow H$ is $\varrho$-strictly pseudononspreading if there exists $\varrho \in[0,1)$ such that

$$
\|T x-T y\|^{2} \leq\|x-y\|^{2}+\varrho\|x-T x-(y-T y)\|^{2}+2\langle x-T x, y-T y\rangle,
$$

for all $x, y \in D(T)$.

Remark 1.5 It is easy to claim that firmly nonexpansive mapping $\Rightarrow$ nonspreading mapping $\Rightarrow \varrho$-strictly pseudononspreading mapping.

Indeed, from the definition of those mappings, $\forall x, y \in C$, we obtain

$$
\begin{gathered}
\|T x-T y\|^{2} \leq\langle x-T x, y-T y\rangle, \quad T \text { is firmly nonexpansive mapping. } \\
\Downarrow \\
\|T x-T y\|^{2} \leq\|x-y\|^{2}+2\langle x-T x, y-T y\rangle, \quad T \text { is nonspreading mapping. } \\
\Downarrow \\
\|T x-T y\|^{2} \leq\|x-y\|^{2}+\varrho\|x-T x-(y-T y)\|^{2}+2\langle x-T x, y-T y\rangle,
\end{gathered}
$$

$T$ is $\varrho$-strictly pseudononspreading mapping.

Clearly, every nonspreading mapping is $\varrho$-strictly pseudononspreading. The following example shows that the class of $\varrho$-strictly pseudononspreading mappings is more general than the class of nonspreading mappings. Let us give an example of a $\varrho$-strictly pseudononspreading mapping satisfying the condition of Definition 1.4.

Example 1.6 Let $X=l^{2}$ with the norm $\|\cdot\|$ defined by

$$
\|x\|=\sqrt{\sum_{i=1}^{\infty} x_{i}^{2}}, \quad \forall x=\left(x_{1}, x_{2}, \ldots, x_{n}, \ldots\right) \in X
$$

$C=\left\{x=\left(x_{1}, x_{2}, \ldots, x_{n}, \ldots\right) \mid x_{i} \in R^{1}, i=1,2, \ldots\right\}$, and let $C$ be an orthogonal subspace of $X$ (i.e., $\forall x, y \in C$, we have $\langle x, y\rangle=0$ ). Then it is obvious that $C$ is a nonempty closed convex subset of $X$. Now, for any $x=\left(x_{1}, x_{2}, \ldots, x_{n}, \ldots\right) \in C$, define a mapping $T: C \rightarrow C$ as follows:

$$
T x= \begin{cases}\left(x_{1}, x_{2}, \ldots, x_{n}, \ldots\right), & \prod_{i=1}^{\infty} x_{i}<0 \\ \left(-2 x_{1},-2 x_{2}, \ldots,-2 x_{n}, \ldots\right), & \prod_{i=1}^{\infty} x_{i} \geq 0\end{cases}
$$

To see that $T$ is $\frac{1}{3}$-strictly pseudononspreading, we break the process of proof into three cases. $\forall x, y \in C$,

Case 1: $\prod_{i=1}^{\infty} x_{i}<0$ and $\prod_{i=1}^{\infty} y_{i}<0$, observe that

$$
\|T x-T y\|^{2} \leq\|x-y\|^{2}+\frac{1}{3}\|x-T x-(y-T y)\|^{2}+2\langle x-T x, y-T y\rangle, \quad \frac{1}{3} \in[0,1),
$$

since $\|T x-T y\|^{2}=\|x-y\|^{2}$ and $\frac{1}{3}\|x-T x-(y-T y)\|^{2}=2\langle x-T x, y-T y\rangle=0$.

Case 2: $\prod_{i=1}^{\infty} x_{i} \leq 0$ and $\prod_{i=1}^{\infty} y_{i} \geq 0$, we obtain $\|T x-T y\|^{2}=\|x+2 y\|^{2}=\|x\|^{2}+4\langle x, y\rangle+$ $4\|y\|^{2}, 2\langle x-T x, y-T y\rangle=0$ and $\frac{1}{3}\|x-T x-(y-T y)\|^{2}=3\|y\|^{2}$. 
Hence,

$$
\begin{aligned}
\| x & -y\left\|^{2}+\frac{1}{3}\right\| x-T x-(y-T y) \|^{2}+2\langle x-T x, y-T y\rangle \\
& =\|x\|^{2}-2\langle x, y\rangle+4\|y\|^{2} \\
& =\|x\|^{2}+4\langle x, y\rangle+4\|y\|^{2}-6\langle x, y\rangle \\
& =\|x\|^{2}+4\langle x, y\rangle+4\|y\|^{2} \quad(\langle x, y\rangle=0) \\
& =\|x+2 y\|^{2}=\|T x-T y\|^{2} .
\end{aligned}
$$

Case 3: $\prod_{i=1}^{\infty} x_{i} \geq 0$ and $\prod_{i=1}^{\infty} y_{i} \geq 0$, we have $\|T x-T y\|^{2}=4\|x-y\|^{2},\|x-T x-(y-T y)\|^{2}=$ $9\|x-y\|^{2}$ and $2\langle x-T x, y-T y\rangle=18\langle x, y\rangle=0$. Thus

$$
\begin{aligned}
\|T x-T y\|^{2} & =4\|x-y\|^{2} \\
& =\|x-y\|^{2}+\frac{1}{3}\|x-T x-(y-T y)\|^{2} \\
& \leq\|x-y\|^{2}+\frac{1}{3}\|x-T x-(y-T y)\|^{2}+2\langle x-T x, y-T y\rangle .
\end{aligned}
$$

From (1), (2) and (3), we obtain that $T$ is $\frac{1}{3}$-strictly pseudononspreading, i.e.,

$$
\|T x-T y\|^{2}=\|x-y\|^{2}+\frac{1}{3}\|x-T x-(y-T y)\|^{2}+2\langle x-T x, y-T y\rangle, \quad \forall x, y \in R .
$$

We can easily know that $F_{i x}(T)=\left\{\left(x_{1}, x_{2}, \ldots, x_{n}, \ldots\right), \prod_{i=1}^{\infty} x_{i}<0\right\} \cup\{0\}$, where $F_{i x}(T)$ is defined by the set of fixed points of $T$.

$T$ is not nonspreading, since for $x=\{0,0, \ldots, 0, \ldots\}, y=\{1,0, \ldots, 0, \ldots\}$, we have $\| T x-$ $T y\left\|^{2}=4,\right\| x-y \|^{2}=1$ and $2\langle x-T x, y-T y\rangle=0$, we obtain

$$
\|T x-T y\|^{2}=4>1=\|x-y\|^{2}+2\langle x-T x, y-T y\rangle .
$$

Since our class of maps contains the class of nonspreading mappings, it also contains the class of firmly nonexpansive mappings.

Remark 1.7 [19] Let $T$ be an $\alpha$-demicontractive mapping on $H$ with $F_{i x}(T) \neq \emptyset$ and $T_{\omega}=$ $(1-\omega) I+\omega T$ for $\omega \in(0, \infty)$ :

(A1) $T \alpha$-demicontractive is equivalent to

$$
\left\langle x-T_{\omega} x, x-q\right\rangle \geq \frac{\omega}{2}\|x-T x\|^{2}, \quad \forall(x, q) \in H \times F_{i x}(T) .
$$

(A2) $F_{i x}(T)=F_{i x}\left(T_{\omega}\right)$ if $\omega \neq 0$.

Remark 1.8 Observe that if $T$ is $\varrho$-strictly pseudononspreading and $F_{i x}(T) \neq \emptyset$, then $\forall x \in$ $D(T)$ and $\forall p \in F_{i x}(T)$, we obtain

$$
\|T x-p\|^{2} \leq\|x-p\|^{2}+\varrho\|x-T x\|^{2}
$$

Thus, every $\varrho$-strictly pseudononspreading mapping with a nonempty fixed point set $F_{i x}(T)$ is demicontractive (see $\left.[20,21]\right)$. 
Remark 1.9 According to Remark 1.7(A1) and the fact that the $\varrho$-strictly pseudononspreading mapping of $T$ is demicontractive, let $I-T_{\omega}=\omega(I-T)$. Then we obtain

$$
\left\langle x-T_{\omega} x, x-q\right\rangle \geq \frac{\omega(1-\varrho)}{2}\|x-T x\|^{2}, \quad \forall(x, q) \in H \times F_{i x}(T) .
$$

In 2011, Osilike and Isiogugu [12] introduced the following propositions and proved a strong convergence theorem somewhat related to a Halpern-type iteration algorithm for a $\varrho$-strictly pseudononspreading mapping in Hilbert spaces.

Proposition 1.10 [12] Let $C$ be a nonempty closed convex subset of a real Hilbert space $H$ and let $T: C \rightarrow C$ be a $\varrho$-strictly pseudononspreading mapping. If $F_{i x}(T) \neq \emptyset$, then it is closed and convex.

Proposition 1.11 [12] Let $C$ be a nonempty closed convex subset of a real Hilbert space $H$ and let $T: C \rightarrow C$ be a $Q$-strictly pseudononspreading mapping. Then $(I-T)$ is demiclosed at 0 .

Theorem 1.12 [12] Let C be a nonempty closed convex subset of a real Hilbert space $H$ and let $T: C \rightarrow C$ be a $\varrho$-strictly pseudononspreading mapping with a nonempty fixed point set $F_{i x}(T)$. Let $\alpha \in[\varrho, 1)$ and let $\left\{\alpha_{n}\right\}_{n=1}^{\infty}$ be a real sequence in $[0,1)$ such that $\lim _{n \rightarrow \infty} \alpha_{n}=0$ and $\sum_{n=0}^{\infty} \alpha_{n}=\infty$. Let $u \in C,\left\{x_{n}\right\}$ and $\left\{z_{n}\right\}$ be sequences in $C$ generated from an arbitrary $x_{1} \in C$ by

$$
\left\{\begin{array}{l}
x_{n+1}=\alpha_{n} u+\left(I-\alpha_{n}\right) z_{n}, \quad n>0 \\
z_{n}=\frac{1}{n} \sum_{k=1}^{n-1} T_{\alpha}^{k} x_{n}, \quad n \geq 1 .
\end{array}\right.
$$

Then $\left\{x_{n}\right\}$ and $\left\{z_{n}\right\}$ converge strongly to $P_{F_{i x}(T)} u$, where $P_{F_{i x}(T)}: H \rightarrow F_{i x}(T)$ is a metric projection of $H$ onto $F_{i x}(T)$.

In 2010, Tian [22] introduced the following theorem for finding an element of a set of solutions to the fixed point of a nonexpansive mapping in a Hilbert space.

Theorem 1.13 [22] Let $f$ be a contraction on a real Hilbert space $H$ and $T$ be a nonexpansive mapping on $H$. Starting with an arbitrary initial $x_{0} \in H$, define a sequence $\left\{x_{n}\right\}$ generated by

$$
x_{n+1}=\alpha_{n} \gamma f\left(x_{n}\right)+\left(I-\mu \alpha_{n} B\right) T x_{n}, \quad n \geq 0,
$$

where $B$ is a $\theta$-Lipschitz and $\eta$-strongly monotone operator on $H$ with $\theta>0, \eta>0$ and $0<$ $\mu<2 \eta / \theta^{2}$. Assume also that a sequence $\left\{\alpha_{n}\right\}$ is a sequence in $(0,1)$ satisfying the following conditions:

(c1) $\lim _{n \rightarrow \infty} \alpha_{n}=0$ and $\sum_{n=0}^{\infty} \alpha_{n}=\infty$,

(c2) $\sum_{n=0}^{\infty}\left|\alpha_{n+1}-\alpha_{n}\right|<\infty$ or $\lim _{n \rightarrow \infty} \alpha_{n+1} / \alpha_{n}=1$.

Then the sequence $\left\{x_{n}\right\}$ generated by (1.8) converges strongly to the unique solution $x^{*} \in$ $F_{i x}(T)$ of the variational inequality

$$
\left\langle(\gamma f-\mu B) x^{*}, x-x^{*}\right\rangle \leq 0, \quad \forall x \in F_{i x}(T) .
$$


In this paper, we combine Theorem 1.12 and Theorem 1.13 and introduce the following general iterative algorithm for a $\varrho$-strictly pseudononspreading mapping $T$.

Algorithm 1.14 Let $x_{0} \in H$ be arbitrary

$$
\left\{\begin{array}{l}
x_{n+1}=\alpha_{n} \gamma f\left(x_{n}\right)+\left(I-\mu \alpha_{n} B\right) z_{n}, \quad n>0 \\
z_{n}=\frac{1}{n} \sum_{k=1}^{n} T_{\alpha}^{k} x_{n}, \quad n \geq 1
\end{array}\right.
$$

where $B: H \rightarrow H$ is $\eta$-strongly monotone and boundedly Lipschitzian, $f$ is an $L$-Lipschitz mapping on $H$ with coefficient $L>0$ and $T_{\alpha}^{k}=(1-\alpha) I+\alpha T^{k}, \alpha \in\left(\varrho_{k}, \frac{1}{2}\right)$.

Under suitable conditions, some strong convergence theorems are proved in the following chapter.

\section{Preliminaries}

Throughout this paper, we write $x_{n} \rightarrow x$ to indicate that the sequence $\left\{x_{n}\right\}$ converges weakly to $x . x_{n} \rightarrow x$ implies that $\left\{x_{n}\right\}$ converges strongly to $x$. The following lemmas are useful for main results.

Definition 2.1 A mapping $T$ is said to be demiclosed if for any sequence $\left\{x_{n}\right\}$ which weakly converges to $y$, and if the sequence $\left\{T x_{n}\right\}$ strongly converges to $z$, then $T(y)=z$.

Lemma 2.2 [3] Assume $\left\{\alpha_{n}\right\}$ is a sequence of nonnegative real numbers such that

$$
\alpha_{n+1} \leq\left(1-\gamma_{n}\right) \alpha_{n}+\delta_{n}, \quad n \geq 0
$$

where $\left\{\gamma_{n}\right\}$ is a sequence in $(0,1)$ and $\left\{\delta_{n}\right\}$ is a sequence in $\mathbb{R}$ such that

(i) $\sum_{n=1}^{\infty} \gamma_{n}=\infty$,

(ii) $\limsup _{n \rightarrow \infty} \frac{\delta_{n}}{\gamma_{n}}=0$ or $\sum_{n=1}^{\infty}\left|\delta_{n}\right|<\infty$.

Then $\lim _{n \rightarrow \infty} \alpha_{n}=0$.

Lemma 2.3 [1] Let $\left\{\mathcal{T}_{n}\right\}$ be a sequence of real numbers that does not decrease at infinity, in the sense that there exists a subsequence $\left\{\mathcal{T}_{n_{j}}\right\}_{j \geq 0}$ of $\left\{\mathcal{T}_{n}\right\}$ which satisfies $\mathcal{T}_{n_{j}}<\mathcal{T}_{n_{j}+1}$ for all $j \geq 0$. Also, consider the sequence of integers $\{\delta(n)\}_{n \geq n_{0}}$ defined by

$$
\delta(n)=\max \left\{k \leq n \mid \mathcal{T}_{k}<\mathcal{T}_{k+1}\right\}
$$

Then $\{\delta(n)\}_{n \geq n_{0}}$ is a nondecreasing sequence verifying $\lim _{n \rightarrow \infty} \delta(n)=\infty, \forall n \geq n_{0}$. It holds that $T_{\delta(n)}<\mathcal{T}_{\delta(n)+1}$, and we have

$$
\mathcal{T}_{n}<\mathcal{T}_{\delta(n)+1}
$$

Lemma 2.4 Let $K$ be a closed convex subset of a real Hilbert space $H$ given $x \in H$ and $y \in K$. Then $y=P_{K} x$ if and only if the following inequality holds:

$$
\langle x-y, y-z\rangle \geq 0, \quad \forall z \in K
$$




\section{Main results}

Let $C$ be a nonempty closed convex subset of a real Hilbert space $H$ and let $T^{k}: C \rightarrow C$ be a $\varrho_{k}$-strictly pseudononspreading mapping with a common nonempty fixed point set $\bigcap_{k}^{n} F_{i x}\left(T^{k}\right)$. Let $f$ be an $L$-Lipschitz mapping on $H$ with coefficient $L>0$. Assume the set $\bigcap_{k}^{n} F_{i x}\left(T^{k}\right)$ is nonempty. Since $\bigcap_{k}^{n} F_{i x}\left(T^{k}\right)$ is closed and convex, the nearest point projection from $C$ onto $\bigcap_{k}^{n} F_{i x}\left(T^{k}\right)$ is well defined. Recall $B: H \rightarrow H$ is $\eta$-strongly monotone and $\theta$-Lipschitzian on $H$ with $\theta>0, \eta>0$. Let $0<\mu<2 \eta / \theta^{2}, 0<\gamma<\mu\left(\eta-\frac{\mu \theta^{2}}{2}\right) / L=\tau / L$, consider the following sequence $\left\{x_{n}\right\}$ defined by

$$
\left\{\begin{array}{l}
x_{n+1}=\alpha_{n} \gamma f\left(x_{n}\right)+\left(I-\mu \alpha_{n} B\right) z_{n}, \quad n>0, \\
z_{n}=\frac{1}{n} \sum_{k=1}^{n} T_{\alpha}^{k} x_{n}, \quad n \geq 1,
\end{array}\right.
$$

where $T_{\alpha}^{k}=(1-\alpha) I+\alpha T^{k}, \alpha \in\left(\varrho_{k}, \frac{1}{2}\right), k=\{1,2, \ldots, n\}$, and $\left\{\alpha_{n}\right\}$ is a sequence in $(0,1)$ satisfying the following conditions:

(c1) $\lim _{n \rightarrow \infty} \alpha_{n}=0$,

(c2) $\sum_{n=0}^{\infty} \alpha_{n}=\infty$ or $\lim _{n \rightarrow \infty} \alpha_{n+1} / \alpha_{n}=1$.

Remark 3.1 [23] Let $H$ be a real Hilbert space. Let $B$ be a $\theta$-Lipschitzian and $\eta$-strongly monotone operator on $H$ with $\theta>0, \eta>0$. Let $0<\mu<2 \eta / \theta^{2}$, let $S=(I-t \mu B)$ and $\mu(\eta-$ $\left.\frac{\mu \theta^{2}}{2}\right)=\tau$, then for $t \in\left(0, \min \left\{1, \frac{1}{\tau}\right\}\right), S$ is a contraction with a constant $1-t \tau$.

Before stating our main result, we introduce some lemmas for algorithm (3.1) as follows.

Lemma 3.2 The sequence $\left\{x_{n}\right\}$ is generated by (3.1) with $T^{k}$ being a $\varrho$-strictly pseudononspreading mapping on $H$ and $\left\{\alpha_{n}\right\} \subset(0,1)$. Then $\left\{x_{n}\right\}$ is bounded.

Proof Let $T_{\alpha}^{k} x=(1-\alpha) x+\alpha T^{k} x$ and $0<\varrho_{k}<\alpha<\frac{1}{2}$. Then $\forall x, y \in C$, we have

$$
\begin{aligned}
\left\|T_{\alpha}^{k} x-T_{\alpha}^{k} y\right\|^{2}= & \alpha\|x-y\|^{2}+(1-\alpha)\left\|T^{k} x-T^{k} y\right\|^{2}-\alpha(1-\alpha)\left\|x-T^{k} x-\left(y-T^{k} y\right)\right\|^{2} \\
\leq & \alpha\|x-y\|^{2}+(1-\alpha)\left[\|x-y\|^{2}+\varrho_{k}\left\|x-T^{k} x-\left(y-T^{k} y\right)\right\|^{2}\right. \\
& \left.+2\left\langle x-T^{k} x, y-T^{k} y\right\rangle\right]-\alpha(1-\alpha)\left\|x-T^{k} x-\left(y-T^{k} y\right)\right\|^{2} \\
= & \|x-y\|^{2}+2(1-\alpha)\left(x-T^{k} x, y-T^{k} y\right\rangle \\
& -(1-\alpha)\left(\alpha-\varrho_{k}\right)\left\|x-T^{k} x-\left(y-T^{k} y\right)\right\|^{2} \\
\leq & \|x-y\|^{2}+2(1-\alpha)\left(x-T^{k} x, y-T^{k} y\right\rangle \\
= & \|x-y\|^{2}+\frac{2(1-\alpha)}{\alpha^{2}}\left\langle x-T_{\alpha}^{k} x, y-T_{\alpha}^{k} y\right\rangle .
\end{aligned}
$$

From $p \in \bigcap_{k}^{n} F_{i x}\left(T^{k}\right)$ and (3.2), we also have

$$
\left\|T_{\alpha}^{k} x_{n}-p\right\| \leq\left\|x_{n}-p\right\|
$$

According to (3.3), (3.1) and Remark 3.1, we obtain

$$
\left\|z_{n}-p\right\|=\left\|\frac{1}{n} \sum_{k=1}^{n} T_{\alpha}^{k} x_{n}-p\right\| \leq \frac{1}{n} \sum_{k=1}^{n}\left\|T_{\alpha}^{k} x_{n}-p\right\| \leq \frac{1}{n} \sum_{k=1}^{n}\left\|x_{n}-p\right\|=\left\|x_{n}-p\right\| .
$$


Thus,

$$
\begin{aligned}
\left\|x_{n+1}-p\right\| & =\left\|\alpha_{n} \gamma\left(f\left(x_{n}\right)-f(p)\right)+\alpha_{n}(\gamma f(p)-\mu B p)+\left(I-\mu \alpha_{n} B\right)\left(z_{n}-p\right)\right\| \\
& \leq \alpha_{n} \gamma\left\|f\left(x_{n}\right)-f(p)\right\|+\alpha_{n}\|\gamma f(p)-\mu B p\|+\left(1-\alpha_{n} \tau\right)\left\|z_{n}-p\right\| \\
& \leq \alpha_{n} \gamma\left\|f\left(x_{n}\right)-f(p)\right\|+\alpha_{n}\|\gamma f(p)-\mu B p\|+\left(1-\alpha_{n} \tau\right)\left\|x_{n}-p\right\|,
\end{aligned}
$$

which combined with $\left\|f\left(x_{n}\right)-f(p)\right\| \leq L\left\|x_{n}-p\right\|$ amounts to

$$
\left\|x_{n+1}-p\right\| \leq\left(1-\alpha_{n}(\tau-\gamma L)\right)\left\|x_{n}-p\right\|+\alpha_{n}\|\gamma f(p)-\mu B p\| .
$$

Putting $M_{1}=\max \left\{\left\|x_{0}-p\right\|,\|\gamma f(p)-\mu B p\|\right\}$, we clearly obtain $\left\|x_{n}-p\right\| \leq M_{1}$. Hence $\left\{x_{n}\right\}_{n=0}^{\infty}$ and $\left\{z_{n}\right\}_{n=1}^{\infty}$ are bounded. From (3.3), we have that $\left\{T_{\alpha}^{k} x_{n}\right\}_{n=1}^{\infty}$ is also bounded.

Now we are in a position to claim the main result.

Theorem 3.3 Assume $C$ is a nonempty closed convex subset of a real Hilbert space $H$ and let $T^{k}: C \rightarrow C$ be a $\varrho_{k}$-strictly pseudononspreading mapping with a common nonempty fixed point set $\bigcap_{k}^{n} F_{i x}\left(T^{k}\right)$. Let $f$ be an L-Lipschitz mapping on $H$ with coefficient $L>0$ and $B: H \rightarrow H$ be $\eta$-strongly monotone and $\theta$-Lipschitzian on $H$ with $\theta>0, \eta>0$. Let $0<\mu<2 \eta / \theta^{2}, 0<\gamma<\mu\left(\eta-\frac{\mu \theta^{2}}{2}\right) / L=\tau / L$. Consider the sequences $\left\{x_{n}\right\}_{n=0}^{\infty}$ and $\left\{z_{n}\right\}_{n=1}^{\infty}$ to be sequences in $C$ generated from an arbitrary $x_{1} \in C$ by (3.1), where $T_{\alpha}^{k}=(1-\alpha) I+\alpha T^{k}$, $\alpha \in\left(\varrho_{k}, \frac{1}{2}\right), k=\{1,2, \ldots, n\},\left\{\alpha_{n}\right\}_{n=1}^{\infty} \in[0,1)$ and $\lim _{n \rightarrow \infty} \alpha_{n}=0$. Then $\left\{x_{n}\right\}_{n=1}^{\infty}$ and $\left\{z_{n}\right\}_{n=1}^{\infty}$ converge strongly to the unique element $x^{*}$ in $\bigcap_{k}^{n} F_{i x}\left(T^{k}\right)$ verifying

$$
P_{\bigcap_{k}^{n} F_{i x}\left(T^{k}\right)}(I-\mu B+\gamma f) x^{*}=x^{*},
$$

which equivalently solves the following variational inequality problem:

$$
x^{*} \in \bigcap_{k}^{n} F_{i x}\left(T^{k}\right), \quad\left\langle(\gamma f-\mu B) x^{*}, v-x^{*}\right\rangle \leq 0, \quad \forall v \in \bigcap_{k}^{n} F_{i x}\left(T^{k}\right) .
$$

Proof According to Lemma 3.2, it is simple to know that $\left\{x_{n}\right\}_{n=0}^{\infty},\left\{z_{n}\right\}_{n=1}^{\infty}$ and $\left\{T_{\alpha}^{k} x_{n}\right\}_{n=1}^{\infty}$ are bounded. Thus, for $\forall y \in C$ and $\forall k=0,1,2, \ldots, n-1$ and according to (3.2) and (3.1), we have

$$
\begin{aligned}
\left\|T_{\alpha}^{k+1} x_{n}-T_{\alpha} y\right\|^{2}= & \left\|T_{\alpha}\left(T_{\alpha}^{k} x_{n}\right)-T_{\alpha} y\right\|^{2} \\
\leq & \left\|T_{\alpha}^{k} x_{n}-y\right\|^{2}+\frac{2}{(1-\alpha)}\left\langle T_{\alpha}^{k} x_{n}-T_{\alpha}^{k+1} x_{n}, y-T_{\alpha} y\right\rangle \\
= & \left\|T_{\alpha}^{k} x_{n}-T_{\alpha} y\right\|^{2}+\left\|T_{\alpha} y-y\right\|^{2}+2\left\langle T_{\alpha}^{k} x_{n}-T_{\alpha} y, T_{\alpha} y-y\right\rangle \\
& +\frac{2}{(1-\alpha)}\left\langle T_{\alpha}^{k} x_{n}-T_{\alpha}^{k+1} x_{n}, y-T_{\alpha} y\right\rangle .
\end{aligned}
$$

Summing (3.9) from $k=0$ to $n$ and dividing by $n$, we obtain

$$
\begin{aligned}
\frac{1}{n}\left\|T_{\alpha}^{k+1} x_{n}-T_{\alpha} y\right\| \leq & \frac{1}{n}\left\|x_{n}-T_{\alpha} y\right\|^{2}+\left\|T_{\alpha} y-y\right\|^{2}+2\left\langle z_{n}-T_{\alpha} y, T_{\alpha} y-y\right\rangle \\
& +\frac{2}{n(1-\alpha)}\left\langle x_{n}-T_{\alpha}^{n} x_{n}, T_{\alpha} y-y\right\rangle .
\end{aligned}
$$


Since $\left\{z_{n}\right\}_{n=1}^{\infty}$ is bounded, then there exists a subsequence $\left\{z_{n j}\right\}_{j=1}^{\infty}$ of $\left\{z_{n}\right\}_{n=1}^{\infty}$ which converges weakly to $\omega \in C$. Replacing $n$ by $n_{j}$ in (3.10), we obtain

$$
\begin{aligned}
\frac{1}{n_{j}}\left\|T_{\alpha}^{k} x_{n_{j}}-T_{\alpha} y\right\|^{2} & \\
\leq & \frac{1}{n_{j}}\left\|x_{n_{j}}-T_{\alpha} y\right\|^{2}+\left\|T_{\alpha} y-y\right\|^{2}+2\left\langle z_{n_{j}}-T_{\alpha} y, T_{\alpha} y-y\right\rangle \\
& \quad \frac{2}{n_{j}(1-\alpha)}\left\langle x_{n_{j}}-T_{\alpha}^{n_{j}} x_{n_{j}}, T_{\alpha} y-y\right\rangle .
\end{aligned}
$$

Since $\left\{x_{n}\right\}_{n=1}^{\infty}$ and $\left\{T_{\alpha}^{n} x_{n}\right\}_{n=1}^{\infty}$ are bounded, letting $j \rightarrow \infty$ in (3.11) yields

$$
0 \leq\left\|T_{\alpha} y-y\right\|^{2}+2\left\langle\omega-T_{\alpha} y, T_{\alpha} y-y\right\rangle \text {. }
$$

Let $y=\omega$ in (3.12). We obtain that $\omega \in F_{i x}\left(T_{\alpha}\right)=F_{i x}(T)$.

Observe that since $\left\{x_{n}\right\}_{n=0}^{\infty}$ and $\left\{z_{n}\right\}_{n=1}^{\infty}$ are bounded, and $\lim _{n \rightarrow \infty} \alpha_{n}=0$, then

$$
\begin{aligned}
\left\|x_{n+1}-z_{n}\right\| & =\alpha_{n}\left\|\gamma f\left(x_{n}\right)-\mu B z_{n}\right\| \\
& \leq \alpha_{n} \gamma\left\|f\left(x_{n}\right)-f(p)\right\|+\alpha_{n}\|\gamma f(p)-\mu B p\|+\alpha_{n}\left\|\mu B\left(z_{n}-p\right)\right\| \\
& \leq \alpha_{n} \gamma L\left\|x_{n}-p\right\|+\alpha_{n}\|\gamma f(p)-\mu B p\|+\alpha_{n} \tau\left\|z_{n}-p\right\|,
\end{aligned}
$$

then

$$
\lim _{n \rightarrow \infty}\left\|x_{n+1}-z_{n}\right\|=0
$$

We next show that

$$
\limsup _{n \rightarrow \infty}\left\{(\gamma f-\mu B) z, x_{n+1}-z\right) \leq 0
$$

Indeed, take $\left\{x_{n_{j}+1}\right\}_{n=1}^{\infty}$ of $\left\{x_{n+1}\right\}_{n=1}^{\infty}$ such that

$$
\limsup _{n \rightarrow \infty}\left\langle(\gamma f-\mu B) x^{*}, x_{n+1}-x^{*}\right\rangle=\lim _{j \rightarrow \infty}\left\langle(\gamma f-\mu B) x^{\prime \prime}, x_{n_{j}+1}-x^{*}\right\rangle
$$

where $x^{*}$ is obtained in (3.7). We may assume that $x_{n_{j}+1} \rightarrow z$ as $j \rightarrow \infty$. From (3.13), we have $z_{n_{j}} \rightarrow z$ as $j \rightarrow \infty$, then to arbitrary bounded linear functional $g$ on $H$, we have

$$
\begin{aligned}
\left\|g\left(z_{n_{j}}\right)-g(z)\right\| & \leq\left\|g\left(z_{n_{j}}\right)-g\left(x_{n_{j}+1}\right)\right\|+\left\|g\left(x_{n_{j}+1}\right)-g(z)\right\| \\
& \leq\|g\|\left\|z_{n_{j}}-x_{n_{j}+1}\right\|+\left\|g\left(x_{n_{j}+1}\right)-g(z)\right\| \\
& \rightarrow 0, \quad \text { as } j \rightarrow 0 .
\end{aligned}
$$

Thus, we obtain $z_{n_{j}} \rightarrow z$ as $j \rightarrow 0$, and $z \in F_{i x}(T)$. Hence, we have

$$
\lim _{j \rightarrow \infty}\left\langle(\gamma f-\mu B) x^{*}, x_{n_{j}+1}-x^{*}\right\rangle=\left\langle(\gamma f-\mu B) x^{*}, z-x^{*}\right\rangle \leq 0 .
$$


Moreover, from (3.1), (3.13) and (3.14), we have

$$
\begin{aligned}
& \limsup _{n \rightarrow \infty}\left\langle(\gamma f-\mu B) z, z_{n}-z\right\rangle \\
& \quad=\limsup _{n \rightarrow \infty}\left\langle(\gamma f-\mu B) z, x_{n+1}-z\right\rangle+\limsup _{n \rightarrow \infty}\left\langle(\gamma f-\mu B) z, z_{n}-x_{n+1}\right\rangle \\
& \quad \leq \limsup _{n \rightarrow \infty}\left\langle(\gamma f-\mu B) z, x_{n+1}-z\right\rangle \leq 0 .
\end{aligned}
$$

As required, finally we show that $x_{n} \rightarrow x^{*}$ and $z_{n} \rightarrow x^{*}$.

According to (3.1), (3.4) and (3.16), we obtain

$$
\begin{aligned}
\left\|x_{n+1}-x^{*}\right\|^{2}= & \left\|\alpha_{n}\left(\gamma f\left(x_{n}\right)-\mu B x^{*}\right)+\left(I-\mu \alpha_{n} B\right) z_{n}-\left(I-\mu \alpha_{n} B\right) x^{*}\right\|^{2} \\
= & \alpha_{n}^{2}\left\|\gamma f\left(x_{n}\right)-\mu B x^{*}\right\|^{2}+\left\|\left(I-\mu \alpha_{n} B\right) z_{n}-\left(I-\mu \alpha_{n} B\right) x^{*}\right\|^{2} \\
& +2 \alpha_{n}\left\langle\left(I-\mu \alpha_{n} B\right) z_{n}-\left(I-\mu \alpha_{n} B\right) x^{*}, \gamma f\left(x_{n}\right)-\mu B x^{*}\right\rangle \\
\leq & \alpha_{n}^{2}\left\|\gamma f\left(x_{n}\right)-\mu B x^{*}\right\|^{2}+\left(1-\alpha_{n} \tau\right)^{2}\left\|z_{n}-x^{*}\right\|^{2} \\
& +2 \alpha_{n}\left[\left\langle z_{n}-x^{*}, \gamma f\left(x_{n}\right)-\mu B x^{*}\right\rangle-\mu \alpha_{n}\left\langle B z_{n}-B x^{*}, \gamma f\left(x_{n}\right)-\mu B x^{*}\right\rangle\right] \\
\leq & {\left[\left(1-\alpha_{n} \tau\right)^{2}+2 \alpha_{n} \gamma L\right]\left\|x_{n}-x^{*}\right\|^{2}+\alpha_{n}\left[2\left\langle z_{n}-x^{*}, \gamma f\left(x_{n}\right)-\mu B x^{*}\right\rangle\right.} \\
& \left.+\alpha_{n}\left\|\gamma f\left(x_{n}\right)-\mu B x^{*}\right\|^{2}+2 \mu \alpha_{n}\left\|B z_{n}-B x^{*}\right\|\left\|\gamma f\left(x_{n}\right)-\mu B x^{*}\right\|\right] \\
\leq & {\left[1-2 \alpha_{n}(\tau-\gamma L)\right]\left\|x_{n}-x^{*}\right\|^{2}+\alpha_{n}\left[2\left\langle x_{n}-x^{*}, \gamma f\left(x_{n}\right)-\mu B x^{*}\right\rangle\right.} \\
& +\alpha_{n}\left\|\gamma f\left(x_{n}\right)-\mu B x^{*}\right\|^{2}+2 \mu \alpha_{n}\left\|B z_{n}-B x^{*}\right\|\left\|\gamma f\left(x_{n}\right)-\mu B x^{*}\right\| \\
& \left.+\alpha_{n} \tau^{2}\left\|x_{n}-x^{*}\right\|^{2}\right] \\
= & \left(1-\bar{\alpha}_{n}\right)\left\|x_{n}-x^{*}\right\|^{2}+\bar{\alpha}_{n} \bar{\beta}_{n},
\end{aligned}
$$

where $\overline{\alpha_{n}}=2 \alpha_{n}(\tau-\gamma L)$,

$$
\begin{aligned}
\bar{\beta}_{n}= & \frac{1}{2(\tau-\gamma L)}\left[2\left\langle x_{n}-x^{*}, \gamma f\left(x_{n}\right)-\mu B x^{*}\right\rangle+\alpha_{n}\left\|\gamma f\left(x_{n}\right)-\mu B x^{*}\right\|^{2}\right. \\
& \left.+2 \mu \alpha_{n}\left\|B z_{n}-B x^{*}\right\|\left\|\gamma f\left(x_{n}\right)-\mu B x^{*}\right\|+\alpha_{n} \tau^{2}\left\|x_{n}-x^{*}\right\|^{2}\right] .
\end{aligned}
$$

It is easily seen that $\lim _{n \rightarrow \infty} \overline{\alpha_{n}}, \sum \bar{\alpha}_{n}=\infty$ and $\limsup _{n \rightarrow \infty} \bar{\beta}_{n} \leq 0$. By Lemma 2.2, we conclude that $x_{n} \rightarrow x^{*}$ as $n \rightarrow \infty$, and $z_{n}$ also converges strongly to the unique element $x^{*}$ in $F_{i x}(T)$. In addition, the variational inequality (3.15) can be written as

$$
\left\langle(I-\mu B+\gamma f) x^{*}-x^{*}, z-x^{*}\right\rangle \geq 0, \quad z \in \bigcap_{k}^{n} F_{i x}\left(T^{k}\right) .
$$

So, by Lemma 2.4, it is equivalent to the fixed point equation

$$
P_{\bigcap_{k}^{n} F_{i x}\left(T^{k}\right)}(I-\mu B+\gamma f) x^{*}=x^{*} .
$$

Remark 3.4 For a nonspreading mapping $T$, we have $\varrho=0$ in Theorem 3.3 to obtain the following corollary. 
Corollary 3.5 Assume $C$ is a nonempty closed convex subset of a real Hilbert space $H$ and let $T^{k}: C \rightarrow C$ be a nonspreading mapping with a common nonempty fixed point set $\bigcap_{k}^{n} F_{i x}\left(T^{k}\right)$. Let $f$ be an L-Lipschitz mapping on $H$ with coefficient $L>0$ and $B: H \rightarrow H$ be $\eta$-strongly monotone and $\theta$-Lipschitzian on $H$ with $\theta>0, \eta>0$. Let $0<\mu<2 \eta / \theta^{2}, 0<$ $\gamma<\mu\left(\eta-\frac{\mu \theta^{2}}{2}\right) / L=\tau / L$, consider the sequences $\left\{x_{n}\right\}_{n=0}^{\infty}$ and $\left\{z_{n}\right\}_{n=1}^{\infty}$ to be sequences in $C$ generated from an arbitrary $x_{1} \in C$ by

$$
\left\{\begin{array}{l}
x_{n+1}=\alpha_{n} \gamma f\left(x_{n}\right)+\left(I-\mu \alpha_{n} B\right) z_{n}, \quad n>0, \\
z_{n}=\frac{1}{n} \sum_{k=1}^{n} T_{\alpha}^{k} x_{n}, \quad n \geq 1,
\end{array}\right.
$$

where $T_{\alpha}^{k}=(1-\alpha) I+\alpha T^{k}, \alpha \in\left(0, \frac{1}{2}\right),\left\{\alpha_{n}\right\}_{n=1}^{\infty} \in[0,1)$ and $\lim _{n \rightarrow \infty} \alpha_{n}=0$. Then $\left\{x_{n}\right\}_{n=1}^{\infty}$ and $\left\{z_{n}\right\}_{n=1}^{\infty}$ converge strongly to the unique element $x^{*}$ in $\bigcap_{k}^{n} F_{i x}\left(T^{k}\right)$ verifying

$$
P_{\bigcap_{k}^{n} F_{i x}\left(T^{k}\right)}(I-\mu B+\gamma f) x^{*}=x^{*}
$$

which equivalently solves the following variational inequality problem:

$$
x^{*} \in \bigcap_{k}^{n} F_{i x}\left(T^{k}\right), \quad\left\langle(\gamma f-\mu B) x^{*}, v-x^{*}\right\rangle \leq 0, \quad \forall v \in \bigcap_{k}^{n} F_{i x}\left(T^{k}\right) .
$$

\section{Competing interests}

The authors declare that they have no competing interests.

\section{Authors' contributions}

All authors contributed equally and significantly in writing this paper. All authors read and approved the final manuscript.

\section{Acknowledgements}

This work is supported in part by the National Natural Science Foundation of China (71272148), the Ph.D. Programs Foundation of Ministry of Education of China (20120032110039) and China Postdoctoral Science Foundation (Grant No. 20100470783)

Received: 12 September 2012 Accepted: 25 January 2013 Published: 28 February 2013

\section{References}

1. Maingé, PE: Strong convergence of projected subgradient methods for nonsmooth and nonstrictly convex minimization. Set-Valued Anal. 16(7-8), 899-912 (2008)

2. Plubtieng, S, Punpaeng, R: A new iterative method for equilibrium problems and fixed point problems of nonexpansive mappings and monotone mappings. Appl. Math. Comput. 197(2), 548-558 (2008)

3. Xu, HK: Iterative algorithms for nonlinear operator. J. Lond. Math. Soc. 66(1), 240-256 (2002)

4. Xu, HK: Viscosity approximation methods for nonexpansive mapping. J. Math. Anal. Appl. 298, 279-291 (2004)

5. Xu, HK: An iterative approach to quadratic optimization. J. Optim. Theory Appl. 116, 659-678 (2003)

6. Yamada, I: Hybrid steepest descent for the variational inequality problems over the intersection of fixed points sets of nonexpansive mapping. In: Butnariu, D, Censor, Y, Reich, S (eds.) Inherently Parallel Algorithms in Feasibility and Optimization and Their Application, pp. 473-504. Elservier, New York (2001)

7. Zeng, LC, Schaible, S, Yao, JC: Iterative algorithm for generalized set-valued strongly nonlinear mixed variational-like inequalities. J. Optim. Theory Appl. 124(3), 725-738 (2005)

8. Browder, FE: Convergence theorems for sequences of nonlinear operators in Banach spaces. Math. Z. 100, 201-225 (1967)

9. Goebel, K, Kirk, WA: Topics in Metric Fixed Point Theory. Cambridge University Press, Cambridge (1990)

10. Goebel, K, Reich, S: Uniform Convexity, Hyperbolic Geometry, and Nonexpansive Mappings. Dekker, New York (1984)

11. Reich, S, Shoikhet, D: Nonlinear Semigroups, Fixed Points, and Geometry of Domains in Banach Spaces. Imperial College Press, London (2005)

12. Osilike, MO, Isiogugu, FO: Weak and strong convergence theorems for nonspreading-type mappings in Hilbert spaces. Nonlinear Anal., Theory Methods Appl. 74(5), 1814-1822 (2011)

13. lemoto, S, Takahashi, W: Approximating common fixed points of nonexpansive mappings and nonspreading mappings in a Hilbert space. Nonlinear Anal. 91, 2080-2089 (2009)

14. Baillon, JB: Un théor'eme de type ergodique pour les contractions non linéaires dans un espace de Hilbert. C. R. Acad. Sci. Paris Sér. A-B 280, A1511-A1514 (1975) 
15. Geobel, K, Kirk, WA: Topics in Metric Fixed Point Theory. Cambridge Stud. Adv. Math., vol. 28, pp. 473-504. Cambridge University Press, Cambridge (1990)

16. Kurokawa, Y, Takahashi, W: Weak and strong convergence theorems for nonspreading mappings in Hilbert spaces. Nonlinear Anal. 73, 1562-1568 (2010)

17. Takahashi, W, Yao, J-C: Fixed point theorems and ergodic theorems for nonlinear mappings in Hilbert spaces. Taiwan. J. Math. 15(2), 457-472 (2011)

18. Browder, FE, Petryshyn, WV: Construction of fixed points of nonlinear mappings in Hilbert spaces. J. Math. Anal. Appl. 20, 197-228 (1967)

19. Maingé, PE: The viscosity approximation process for quasi-nonexpansive mappings in Hilbert spaces. Comput. Math. Appl. 59, 74-79 (2010)

20. Hicks, TL, Kubicek, JR: On the Mann iterative process in Hilbert spaces. J. Math. Anal. Appl. 59, 498-504 (1977)

21. Naimpally, SA, Singh, KL: Extensions of some fixed point theorems of Rhoades. J. Math. Anal. Appl. 96, 437-446 (1983)

22. Ming, T: A general iterative algorithm for nonexpansive mappings in Hilbert space. Nonlinear Anal. 73, 689-694 (2010)

23. Deng, BC, Chen, T, Li, ZF: Cyclic iterative method for strictly pseudononspreading in Hilbert space. J. Appl. Math. 2012, Article ID 435676 (2012). doi:10.1155/2012/435676

doi:10.1186/1029-242X-2013-80

Cite this article as: Deng et al.: Viscosity iteration algorithm for a $\varrho$-strictly pseudononspreading mapping in a Hilbert space. Journal of Inequalities and Applications 2013 2013:80.

\section{Submit your manuscript to a SpringerOpen ${ }^{\ominus}$ journal and benefit from:}

- Convenient online submission

Rigorous peer review

- Immediate publication on acceptance

- Open access: articles freely available online

- High visibility within the field

- Retaining the copyright to your article 